\title{
On some Gelfand-Mazur like theorems in Banach algebras
}

\section{V.K. Srinivasan}

The following Gelfand-Mazur like theorems are proved in this paper :

(1) A complex Banach algebra which is locally finite, and which is also an integral domain, is isomorphic to the complex field $\mathbf{0}$.

(2) A complex Banach algebra which is a noetherian domain is isomorphic to $\mathbb{a}$.

(3) A complex Banach algebra which is a principal ideal domain is isomorphic to $\mathbb{a}$.

An application is given to the algebra of all complex formal power series in several variables.

\section{Introduction}

A Gelfand-Mazur like theorem is a theorem which asserts that a complex Banach algebra is isomorphic to the complex field, if either an algebraic condition or an analytical condition is imposed on the algebra. Sinclair and Tullo [4] have shown that a Banach algebra which is a noetherian ring is finite dimensional as a linear space. In this paper, after establishing a preliminary Gelfand-Mazur like theorem, which requires a local finiteness condition, we prove a stronger result, which asserts that a complex Banach algebra wich is a noetherian integral domain is necessarily isomorphic to c . Apart from strengthening the result of Sinclair and Tullo [4], the above result answers a few of the open problems listed in the paper of

Received 6 February 1979. 
Srinivasan and Hu Shaing [5]. An important consequence is the result which asserts that a complex Banach algebra which is a principal ideal domain is isomorphic to the field $\mathbb{d}$ of complex numbers. The above result was proved by a totally different method in [5].

In Section 2, some of the relevant definitions and main theorems used in the sequel are gathered. The main results are proved in Section 3.

\section{2.}

The results involving the theory of Banach algebras are from the book of Rickart [3].

DEFINITION 2.1. Let $A$ be a complex Banach algebra, with the identity element denoted by $1, A$ being not necessarily commutative. An element $a \in A$ is called a left topological zero divisor, if there exists a sequence $\left\{z_{n}\right\}, z_{n} \in A$, with $\left\|z_{n}\right\|=1$ for all $n$ and $\left\|a z_{n}\right\| \rightarrow 0$ as $n+\infty$. A right topological zero divisor is defined similarly. By a topological zero divisor we mean either a left topological zero divisor or a right topological zero divisor.

THEOREM 2.1. Let $A$ be a complex Banach algebra with an identity element. If $A$ has no topological zero divisors other than the element $0 \in A$, then $A$ is isomorphic to the complex field $\mathbb{a}$.

The next theorem is stated for the specific case of integral domains. An integral domain is a commutative ring with an identity element $1 \neq 0$, having no divisors of zero. The proof of the next theorem uses Theorem 2.1 and a lemma stated in Rickart ([3], Lemma 1.5.2, p. 21).

THEOREM 2.2. Let $A$ be a complex Banach algebra which as a ring is an integral domain. The following relations hold.

- (i) Let $h \neq 0, h \in A$. The necessary and sufficient condition for $h$ not to be a topological zero divisor is that the principal ideal ( $h$ ) be closed in $A$.

(ii) If for each $h \neq 0, h \in A$, the ideal (h) is closed, then $A$ is isomorphic to $\mathbb{a}$.

DEFINITION 2.2. Let $A$ be a linear algebra over $\mathbb{d}$. $A$ is said to be locally finite if every subalgebra of $A$ which is finitely generated is 
finite dimensional as a linear subspace.

For results on the ring of formal power series the reader is referred to Northcott ([2], p. 108).

THEOREM 2.3 ([2], [1]). Let $K$ be any commutative field. Let $K\left(x_{1}, x_{2}, \ldots, x_{n}\right)$ be the ring of all formal power series in the indeterminates $x_{1}, x_{2}, \ldots, x_{n}, K\left(x_{1}, x_{2}, \ldots, x_{n}\right)$ is a noetherian domain and also a unique factorization domain.

The next theorem is the key tool used in Section 3.

THEOREM 2.4 (Sinclair and Tullo [4]). Let $A$ be a complex Banach algebra which is noetherian. Then $A$ is finite dimensional.

3 .

The results stated in the introduction are proved in this section.

THEOREM 3.1. Let $A$ be a complex Banach algebra which is also an integral domain. If $A$ is locally finite, then $A$ is isomorphic to the fiezd a of complex numbers.

Proof. In view of Theorem 2.2 (ii), it suffices to show that for each $h \neq 0, h \in A$, the principal ideal $(h)$ is closed. Since $A$ is locally finite and $(h)$ is finitely generated as a subalgebra, it follows that (h) is finite dimensional as a subspace. Since all finite dimensional subspaces of a Banach space are closed, $(h)$ is closed. The result now follows.

THEOREM 3.2. Let $\mathbb{a}^{n}(n \geq 2)$ be the complex linear space of $n$-tuples. It can not be simultaneously made into a normed algebra and an integral domain.

Proof. We first note that any norm on $\boldsymbol{\sigma}^{n}$ is automatically complete. If $\mathbb{a}^{n} \quad(n \geq 2)$ can be simultaneously made into a normed algebra and an integral domain, then it would become a Banach algebra which would be locally finite. By Theorem 3.1 it would be isomorphic to $\mathbb{a}$, which is impossible. Hence the result follows.

Srinivasan and Hu Shaing in their paper [5] raised the question whether a complex Banach algebra which is a noetherian domain, is 
isomorphic to $\mathbb{a}$. This is answered in the affirmative in the next theorem, which further strengthens the existing result of Theorem 2.4 .

THEOREM 3.3. Let $A$ be a complex Banach algebra which is also a noetherian domain. Then $A$ is isomorphic to $\mathbb{I}$.

Proof. The result follows by combining Theorem 2.4 with Theorem 3.1 (or Theorem 3.2).

The next theorem was proved by Srinivasan and Hu Shaing [5] by a totally different method. Since every principal ideal domain is also a noetherian domain, the proof of the next theorem follows from Theorem 3.3.

THEOREM 3.4 ([5]). Let $A$ be a complex Banach algebra, which is also a principal ideal domain. Then $A$ is isomorphic to $\mathbb{b}$.

Srinivasan and Hu Shaing [5] raised the question whether the algebra $\mathbb{a}\left(x_{1}, x_{2}, \ldots, x_{n}\right)$, of all complex formal power series in several variables, can be made into a Banach algebra under a suitable norm. The answer is in the negative, as is shown in the next theorem.

THEOREM 3.5. Let $\mathbb{a}\left(x_{1}, x_{2}, \ldots, x_{n}\right)$ be the algebra of all complex formal power series in several variables, taken under the usual operations. Let $P\left(x_{1}, x_{2}, \ldots, x_{n}\right)$ be the algebra of all complex polynomials in several variables taken under the usual operations. Neither of them can be made into Banach algebras under any norm.

Proof. Both of them are noetherian domains (Theorem 2.3) and are also algebras. The result follows from Theorem 3.3. (It also can be deduced directly from Theorem 2.4, since both the spaces are infinite dimensional.)

\section{References}

[1] I.S. Cohen, "On the structure and ideal theory of complete local rings", Trans. Amer. Math. Soc. 59 (1946), 54-106.

[2] D.G. Northcott, Ideal theory (Cambridge Tracts in Mathematics and Mathematical Physics, 42. Cambridge University Press, Cambridge, 1960). 
[3] Charles E. Rickart, General theory of Banach algebras (Van Nostrand, Princeton, New Jersey; Toronto; London; New York; 1960).

[4] Allan M. Sinclair and Alan W. Tullo, "Noetherian Banach algebras are finite dimensional", Math. Ann. 211 (1974), 151-153.

[5] V.K. Srinivasan and Hu Shaing, "Algebraic conditions in Banach algebras", submitted. See also the abstract, "Banach algebras and Bezout domains", 78T-BI80 in Notices Amer. Math. Soc. 25 (1978), A-590.

Department of Mathematics, University of Texas at EI Paso, El Paso,

Texas, USA. 